\title{
NOUVELle
}

\section{La génétique établit le lien entre plusieurs maladies cardiovasculaires affectant préférentiellement les femmes}

Adrien Georges ${ }^{1,2}$, Nabila Bouatia-Naji1,2

\footnotetext{
${ }^{1}$ Inserm, U970, Paris cardiovascular research center - PARCC, 56, rue Leblanc, 75737 Paris Cedex 15, France.

${ }^{2}$ Université Paris Descartes, Sorbonne Paris Cité, UMR-S970, 56, rue Leblanc, 75737 Paris Cedex 15, France. nabila.bouatia-naji@inserm.fr
}

> La dissection spontanée de l'artère coronaire (SCAD pour spontaneous coronary artery dissection) est une cause méconnue et encore peu étudiée d'infarctus du myocarde [1]. Cette affection touche principalement les femmes, sans distinction d'âge, et représente de 23 à $36 \%$ des infarctus du myocarde chez les femmes de moins de 50 ans. Le SCAD est également une cause importante d'infarctus associé à la grossesse. C'est un événement aigu causé par la dissociation d'une partie de la paroi de l'artère coronaire, créant un hématome intra-mural comprimant la lumière de l'artère et pouvant déboucher sur son occlusion totale et un infarctus du myocarde.

La prise en compte du SCAD parmi les causes possibles d'un infarctus du myocarde est relativement récente, et cette affection présente des spécificités cliniques et de prise en charge qui diffèrent de celle d'un infarctus classique, dont la prévalence est moindre chez les femmes. Les mécanismes cellulaires et moléculaires amenant au SCAD sont mal connus. Le SCAD n'est pas associé aux facteurs de risque habituels des maladies cardiovasculaires, comme l’âge, une prévalence élevée d'athérosclérose, la dyslipidémie, un indice de masse corporelle élevé ou la consommation de tabac [2].

Plusieurs cas de SCAD survenus chez plusieurs membres d'une même famille ont été décrits, généralement des paires sœur-sœur ou mère-enfant, suggérant une origine au moins partiellement génétique de cette maladie. Cependant, la recherche de mutations causales ciblant des gènes impliqués dans divers syndromes des tissus conjonctifs n'a pas permis d'identifier de candidats robustes pouvant être associés au SCAD [1].

Des investigations sur les artères rénales et carotides chez des patientes et patients atteints de SCAD ont montré la présence de lésions de dysplasie fibromusculaire (DFM) chez 17 à $86 \%$ d'entre eux, suggérant une forte association de ces deux affections [1]. La DFM présente un profil épidémiologique similaire au SCAD, affectant une large majorité de femmes sans association avec les facteurs de risque de l'athérosclérose [3]. La DFM se manifeste par des déformations non-athéromateuses et non-inflammatoires de la paroi des artères de moyen calibre [3]. Quelques cas de DFM sur des artères coronaires ayant subi une dissection ont également été décrits [2]. Cependant, la majorité des cas de DFM connus concernent les artères rénales, vertébrales ou carotides et sont découverts au cours d'investigations poussées dans le cadre d'une hypertension artérielle secondaire ou à la suite d'accidents vasculaires cérébraux. Plusieurs études ont suggéré que la DFM était sous-diagnostiquée, souvent asymptomatique, et on estime qu'elle pourrait affecter jusqu'à $6 \%$ des individus de la population générale.
Nous avons récemment découvert le premier variant génétique associé à la DFM, nommé rs9349379 [4]. II s'agit d'un variant génétique commun, situé à proximité du gène PHACTRI (phosphatase and actin regulator 1 ) sur le chromosome 6. Nous avons observé que, dans la population étudiée, l'allèle $A$ du variant confère une augmentation de $40 \%$ du risque de DFM. Soulignons que ce même variant est également associé à d'autres maladies ou problèmes cardiovasculaires. L'allèle $A$ confère en effet un risque accru de dissection des artères cervicales (CeAD) [5], alors que l'allèle $G$ confère notamment un risque accru d'infarctus du myocarde [6]. Ces observations étonnantes nous ont conduit à rechercher si ce variant pouvait également être associé au SCAD, et quel serait l'allèle à risque dans ce cas.

Pour répondre à ces questions, nous avons déterminé le génotype du variant rs9349379 chez 1055 patients souffrant de SCAD, issus de quatre cohortes différentes et de trois pays (France, Royaume-Uni, États-Unis), chaque cohorte étant comparée à une cohorte témoin issue du même pays [7].

Il est remarquable de constater que chacune des 4 études cas-témoins montre un net enrichissement de l'allèle A du variant rs9349379 chez les patients souffrant de SCAD (Figure 1 , premier panneau). La fréquence observée de l'allèle $A$ se situe entre $68 \%$ et $76 \%$ en fonction des cohortes, alors 


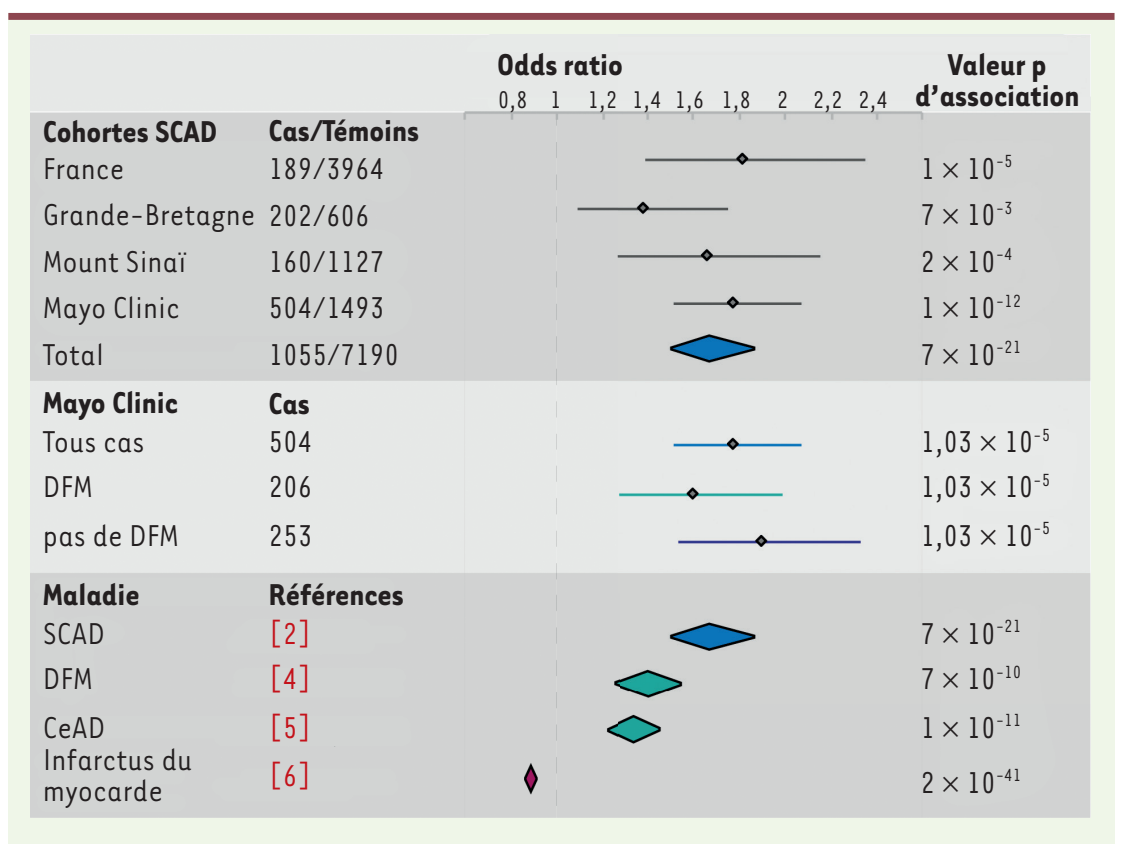

Figure 1. Association du variant rs9349379 avec le SCAD et plusieurs autres maladies. Le risque relatif ou odds ratio calculé pour l'allèle A du variant rs 9349379 est représenté dans les différentes cohortes de patients présentant un SCAD (Dissection spontanée de l'artère coronaire), ainsi que dans les méta-analyses de patients victimes de SCAD, DFM (dysplasie fibromusculaire), CeAD (dissection des artères cervicales), ou ayant subi un infarctus du myocarde. Les barres et les losanges représentent l'intervalle de confiance à $95 \%$, respectivement dans le cas des cohortes SCAD et des méta-analyses. La valeur $p$ d'association est indiquée pour chaque cas.

que la fréquence de cet allèle dans les cohortes témoins se situe entre $59 \%$ et $63 \%$. Sur l'ensemble des quatre cohortes, la fréquence de rs 9349379 A, de $61 \%$ chez les témoins, passe à $72 \%$ chez les patients victimes de SCAD.

Afin de déterminer si ces observations montraient une association statistiquement significative du variant rs9349379 avec le SCAD, les données ont été modélisées à l'aide d'une régression logistique avec un modèle additif. Ce modèle repose sur l'hypothèse que chaque allèle, pris indépendamment, peut conférer ou non un risque. Cette modélisation montre une association statistiquement significative $(p<0,01)$ du génotype de rs9349379 avec la maladie dans chacune des quatre cohortes. Sur l'ensemble des 4 cohortes, la valeur $p$ observée est de $7 \times 10^{-21}$, très en deçà du seuil de significativité estimé pour les études d'association à l'échelle du génome $\left(5 \times 10^{-8}\right)$. Le rapport des chances $^{1}$ (odds ratio) est estimé par notre étude comme ayant $95 \%$ de probabilité de se situer entre 1,5 et 1,86. Cela signifie que l'allèle $A$ de rs9349379 confèrerait une augmentation de 50 à $86 \%$ du risque de SCAD. Étant donné qu'une proportion importante des patients souffrant de SCAD présente également des lésions de DFM, nous avons voulu vérifier si une part de l'association avec le variant rs9349379 pouvait être due à la présence de patients DFM dans ces cohortes (Figure l, second panneau). Nous avons donc analysé séparément la fréquence de l'allèle rs9349379-A chez des patients SCAD présentant des lésions de DFM et chez les patients SCAD ne présentant pas de lésion DFM détectable, au sein de la cohorte de

\footnotetext{
1 L'odds ratio ou risque relatif rapproché est une mesure statistique exprimant le degré de dépendance entre des variables aléatoires qualitatives.
}

patients de la clinique Mayo. L'odds ratio a été estimé comme étant supérieur chez les patients ne présentant pas de lésion DFM (intervalle de confiance à $95 \%$ entre 1,53 et 2,33) par comparaison aux patients en présentant (intervalle de confiance à $95 \%$ entre 1,28 et 1,99$)$. Le recouvrement des intervalles de confiance ne permet pas d'affirmer avec certitude que l'allèle rs9349379-A procure un risque plus important de SCAD que de DFM. Cependant, cette stratification démontre que l'association du variant rs9349379 avec le SCAD est indépendante de la présence (connue ou non) de patients DFM dans les cohortes de patients SCAD.

Cette découverte confirme l'existence de similarités, au niveau génétique, entre les différentes pathologies artérielles non-athéromateuses que sont le SCAD, la DFM et la CeAD (Figure 1, troisième panneau). À l'inverse, cette association du SCAD à l'allèle rs9349379-A souligne les différences avec l'infarctus du myocarde classique, généralement d'origine athéromateuse, dont le risque est augmenté par l'allèle rs9349379-G.

Plusieurs hypothèses existent sur la fonction moléculaire affectée par ce variant, situé dans une région noncodante du génome et qui semble jouer un rôle de régulation de la transcription (Figure 2). Le gène PHACTR1 possède des régions promotrices situées légèrement en aval du variant rs9349379, et son expression est fortement corrélée au génotype de rs9349379 dans les artères, l'allèle A étant associé à une expression plus élevée du gène [8]. PHACTRI code une protéine qui contrôle la polymérisation de l'actine et la formation de fibres de stress, et qui a été proposée comme jouant un rôle dans la migration cellulaire et l'angiogenèse.

Cependant, une étude récente a suggéré, à l'aide de modifications de cellules pluripotentes par la méthode CRISPR-Cas9, que le variant rs9349379 


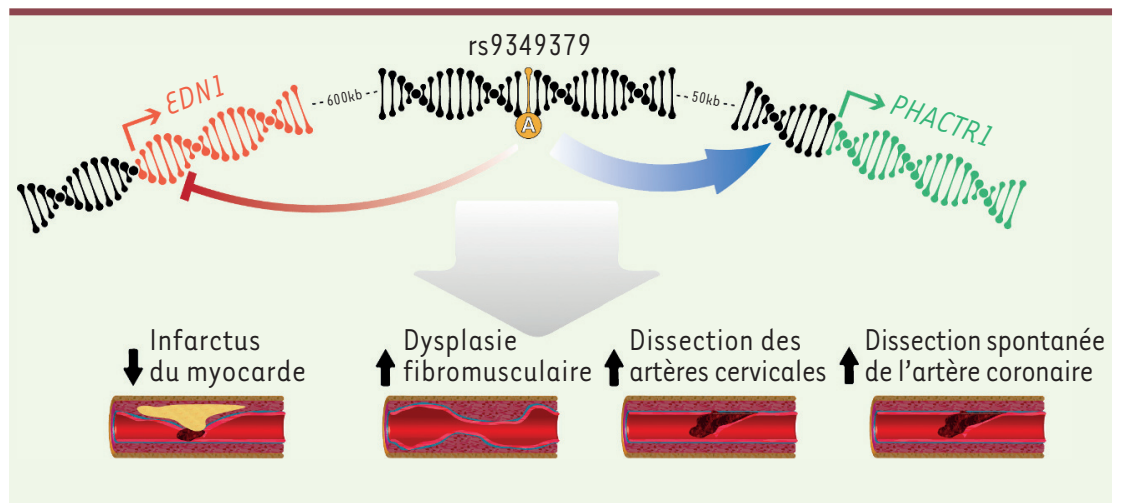

Figure 2. Illustration des maladies et mécanismes moléculaires associés au variant génétique rs9349379. L'allèle A du variant rs9349379 semble associé à une plus grande expression (flèche bleue) du gène PHACTRI (phosphatase and actin regulator 1), situé en aval du variant, et à une plus faible expression (flèche-arrêt rouge) du gène EDNI (endothelin-1), situé en amont du variant. Ces mécanismes, peut-être associés à d'autres non encore connus, mènent à un risque réduit d'infarctus du myocarde, et à des risques augmentés de DFM (dysplasie fibromusculaire), CeAD (dissection des artères cervicales) ou SCAD (dissection spontanée de l'artère coronaire).

pouvait également affecter l'expression du gène $E D N I$ (endothelin 1), situé 600 kilobases en amont du variant, et qui code le peptide précuseur de l'endothéline 1 , une hormone jouant un rôle clé dans la contraction des vaisseaux sanguins et la prolifération $(\rightarrow)$ Voir la Synthèse de F. Pinet $\mathrm{m} / \mathrm{s} \mathrm{n}^{\circ} 3$, des cellules vascu- mars 2004, page 339 laires $[9,10](\rightarrow)$.

L'allèle $A$ serait alors associé à une expression plus faible d'EDN1, observation cohérente avec le dosage plasmatique de l'endothéline 1 dans une cohorte d'individus sains. Nous avons également observé une corrélation entre le génotype de rs9349379 et le dosage plasmatique de l'endothéline $1 \mathrm{chez}$ une partie des individus souffrant de SCAD, issus de la cohorte britannique [7]. Cependant, une étude similaire a pondéré ces résultats en ne retrouvant pas l'effet du génotype de rs 9349379 sur l'expression du gène EDNI [11].
II est possible que plusieurs mécanismes entrent en jeu, et de plus amples recherches seront nécessaires pour mieux comprendre le rôle de ce locus. Plus largement, notre étude montre que le SCAD relève d'un mécanisme d'hérédité complexe, dans lequel la combinaison d'un certain nombre de variants rares ou fréquents procure un risque accru ou réduit de développer la maladie. Cette étude suggère également que des mécanismes moléculaires communs peuvent participer à différentes maladies cardiovasculaires non-athéromateuses. L'exploration de ces mécanismes représente un enjeu majeur d'une prise en charge personnalisée, avec notamment une meilleure prise en compte du sexe, des patients atteints de maladies cardiovasculaires. $\diamond$

Genetics provides a link between cardiovascular diseases predominantly afflicting women

\section{LIENS D'INTÉRÊT}

Les auteurs déclarent n'avoir aucun lien d'intérêt concernant les données publiées dans cet article.

\section{RÉFÉRENCES}

1. Hayes SN, Kim ESH, Saw J, et al. Spontaneous coronary artery dissection: current state of the science. A scientific statement from the american heart association. Circulation 2018; 137 : e523-57.

2. Adlam D, Alfonso F, Maas A, et al. European society of cardiology, acute cardiovascular care association, SCAD study group: a position paper on spontaneous coronary artery dissection. Eur Heart J 2018 ; 39 : 3353-68

3. Persu A, Van der Niepen P, Touzé $\varepsilon$, et al. Revisiting fibromuscular dysplasia: rationale of the european fibromuscular dysplasia initiative. Hypertension $2016 ; 68: 832-9$

4. Kiando SR, Tucker NR, Castro-Vega LJ, et al. PHACTRI is a genetic susceptibility locus for fibromuscular dysplasia supporting its complex genetic pattern of inheritance. PLoS Genet 2016 ; 12 : el006367.

5. Debette S, Kamatani Y, Metso TM, et al. Common variation in PHACTRl is associated with susceptibility to cervical artery dissection. Nat Genet 2015 ; 47 : 78-83.

6. Nikpay M, Goel A, Won HH, et al. A comprehensive 1,000 genomes-based genome-wide association meta-analysis of coronary artery disease. Nat Genet $2015 ; 47$ : 1121-30.

7. Adlam D, Olson TM, Combaret N, et al. Association of the PHACTRl/EDNl genetic locus with spontaneous coronary artery dissection. J Am Coll Cardiol 2019; $73: 58-66$.

8. Codina-Fauteux VA, Beaudoin M, Lalonde S, et al. PHACTRl splicing isoforms and eQTLs in atherosclerosis-relevant human cells. BMC Med Genet $2018 ; 19: 97$

9. Gupta RM, Hadaya J, Trehan A, et al. A genetic variant associated with five vascular diseases is a distal regulator of endothelin-1 gene expression. Cell 2017 ; $170: 522-33$

10. Pinet F. À quoi sert le système endothéline? Med Sci (Paris) $2004 ; 20: 339-45$.

11. Wang $X$, Musunuru K. Confirmation of causal rs9349379-PHACTRl expression quantitative trait locus in human-induced pluripotent stem cell endothelial cells. Circ Genom Precis Med 2018 ; 11 : e002327.
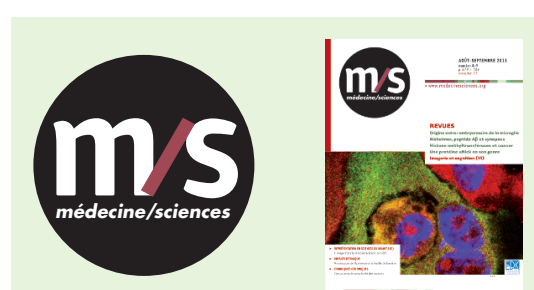

Abonnez-vous

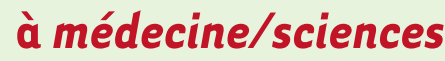

Bulletin d'abonnement page 714 dans ce numéro de $\mathrm{m} / \mathrm{s}$ la filière de santé neuromusculaire FILNEMUS

www. filnemus.fr

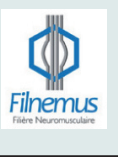

\section{Retrouvez toutes les Actualités de la Myologie sur les sites de:}

la Société Française de Myologie www.sfmyologie.org

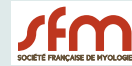

wwitilnemus. fr 